\title{
Implementación de sistemas de evaluación de personal en empresas chilenas, etapa inicial para medir su impacto en la gestión de la empresa
}

\section{Implementation of personnel evaluation systems in Chilean companies, initial stage to measure their impact on the management of the company}

\author{
Jorge Sánchez Henríquez, Sandra Alvear Vega* \\ Universidad de Talca, Chile
}

Recibido el 1 de marzo de 2018; aceptado el 1 de enero de 2020

Disponible en Internet el: 6 de octubre de 2020

\section{Resumen}

La implementación de sistemas de evaluación del desempeño cada vez requiere de mayor revisión, en este aspecto existe una falta de auditoría de recursos humanos en las organizaciones, en esta área específica. El objetivo principal de este trabajo fue reducir la dimensionalidad de las variables que influyen en la etapa de "implementación" del proceso de medición del desempeño de los trabajadores. Es un estudio analítico, observacional de corte transversal, que incluyó la evaluación de 22 variables y la aplicación de 61 procedimientos de auditoría específicos en 116 empresas, públicas y privadas, de diferentes rubros. Para el análisis se aplicó técnicas de análisis factorial exploratorio. Los resultados muestran que los factores en la implementación del proceso de evaluación del desempeño de los trabajadores en el presente estudio son Información, Evaluación, Capacitación, Apelación, Retroalimentación y Aplicación. Dichas componentes son una fuente inicial para la construcción de un índice que permita medir el impacto de la evaluación de desempeño en la gestión de la empresa.

\section{Código JEL: M12, J24, M42}

Palabras clave: Auditoria de recursos humanos; Evaluación del desempeño e implementación de la evaluación del desempeño

\footnotetext{
*Autor para correspondencia

Correo electrónico salvear@utalca.cl (S. Alvear Vega).

La revisión por pares es responsabilidad de la Universidad Nacional Autónoma de México. 


\begin{abstract}
The implementation of performance evaluation systems requires a permanent review; In this regard, the lack of audit of human resources in organizations affects this specific area.

The main objective of this work was to identify the variables that influence the stage of implementation of the process of measuring the performance of workers. It is an analytical, observational cross-sectional study, which was conducted using a specific evaluation' instrument to evaluate the implementation stage of the performance evaluation system, which included the evaluation of 22 variables and the application of 61 specific audit procedures and that It was carried out in 116 companies, public and private, of different industries.

For the exploratory factor analysis were applied. The principal factors that influence the process of implementing the process of evaluating the performance of workers in the present study are; Information, Evaluation, Training, Appeal, Feedback and Application. These components are the initial source for the construction of an index that allows measuring the impact of performance evaluation in the management of the company.
\end{abstract}

JEL codes: M12, J24, M42

Keywords: Human resource auditing; Performance evaluation and implementation of performance evaluation

\title{
Introducción
}

Tema de la evaluación del desempeño de los trabajadores en las empresas es algo que tanto las empresas como los trabajadores consideran de suma importancia, en efecto, autores como Milkovich y Boudreau (1994), Werther y Davis (2013), Ariza, Morales y Morales (2004), Ulrich y Brockbank (2007), Luecke (2007), Dolan, Valle, Jackson y Schuler (2007), Sánchez (2014), Chiavenato (2017) plantean claramente la relevancia de la evaluación del desempeño para la organización y la importancia de realizarla como un proceso sistémico. Siempre será necesario de las empresas evalúen el desempeño de su personal, en efecto, Sastre y Aguilar (2003), Ramlall (2006), Wright, Dunford y Snell (2001). Kliksberg (1998), Gruman y Saks (2011), Valle (2004) y Bock (2015), afirman que las organizaciones deben evaluar el desempeño de sus trabajadores si quieren asegurarse realmente de cumplir con sus objetivos estratégicos. El logro de los objetivos estratégicos de las organizaciones está directamente relacionado con el desempeño del personal, las empresas hoy necesitan información relevante que debería ser proporcionada por sus sistemas de evaluación del desempeño, uno de los motivos más importantes por el que las organizaciones deben implementar un sistema de evaluación y control de gestión de su personal, es para saber si sus trabajadores están efectivamente contribuyendo al logro de los objetivos. Sánchez y Alvear (2018) explicitan la necesidad de 
realizar una adecuada planificación del sistema de evaluación del desempeño, en especial en la etapa de implementación del mismo, en ese sentido Manjarrés, Castell y Luna (2013) y Kehoe y Wright (2013), plantean que la evaluación del desempeño tiene que ir alineada con el direccionamiento estratégico de la empresa, con los procesos organizacionales y con los objetivos e indicadores que se quieren lograr a lo largo de la operación organizativa. Para Sánchez y Calderón (2012), Parent, Sloan y Tsuchida (2015) y Sánchez y Ramirez (2017) el desempeño actual y sobretodo el desempeño potencial y futuro de los trabajadores en el futuro, debe ser una de las variables más relevantes en la dirección de personas.

El problema detectado radica en que la etapa de implementación de los sistemas de evaluación del desempeño en las organizaciones, es en general, algo muy poco estudiada, esto justifica la realización de este trabajo, en efecto, se requiere cada vez más, de la realización de auditorías de recursos humanos, Sánchez y Rojas (2014). Es fundamental que en la etapa de planificación del sistema de evaluación se consideren todos los aspectos relacionados con las variables que pueden afectar su implementación, Sánchez y Alvear (2018). El objetivo principal de la investigación fue identificar las variables que influyen en la implementación del proceso de medición del desempeño de los trabajadores, para lograrlo se realizaron auditorías de recursos humanos específicas en la etapa de implementación de los procesos de evaluación del personal, en organizaciones de diferente tipo, giro y tamaño. La evidencia encontrada en las auditorías realizadas en 116 organizaciones chilenas, demostraron que no hubo ninguna de ellas que cumplieran a cabalidad con lo requerido para tener una buena etapa de implementación de sus sistemas de evaluación. Es necesario seguir trabajando en este aspecto y se propone que para futuras investigaciones se centre el trabajo en solo 6 variables.

\section{Marco teórico}

\section{Evaluación del desempeño}

Hay diferentes visiones al momento de enfrentar el tema del desempeño y su evaluación, por ejemplo Dessler y Varela (2011) definen la evaluación como la valoración del desempeño actual o anterior de un trabajador en comparación con estándares, en el mismo sentido Mondy y Noe (2005) lo asocian a un sistema de revisión y evaluación del desempeño laboral individual o de equipos, para Pereda y Berrocal (2011), la evaluación del desempeño es el proceso sistemático y periódico de medida objetiva del nivel de eficacia y eficiencia de un empleado, o equipo, en su trabajo, Gómez-Mejía, Balkin y Cardy (2008) complementan diciendo que la valoración del rendimiento implica identificar, medir y gestionar el rendimiento de las personas de una organización. Por su parte Sastre y Aguilar (2003) postulan que es un proceso sistemático y estructurado, de seguimiento de la labor profesional del empleado, para valorar su actuación y los resultados logrados en el desempeño de su cargo, lo mismo plantean 
Fombrun, Tichy y Devanna (1984), para Salgado y Cabal (2011) la evaluación del desempeño tiene el propósito de valorar de forma sistemática y objetiva, el rendimiento o desempeño de los empleados en la organización. Es el grado en que el empleado cumple con los requisitos de su cargo (Trillo, 2001) o el modo en que un trabajador realiza las funciones y tareas que tiene asignadas, (Salgado y Cabal, 2011), por su parte Pérez, Leyva, Bajuelo y Pérez (2015), Alles (2014) y Valdés, Garza, Pérez, Gé y Chávez (2015) complementan diciendo que la evaluación del desempeño debe estar de acuerdo con la misión y los objetivos fijados por la empresa y las decisiones relacionadas a su cumplimiento, y demuestra en el ejercicio de sus funciones poseer las competencias exigidas para el puesto de trabajo que ocupa el trabajador, por su parte Sánchez y Bustamante (2008), lo relacionan con valor agregado y plantean que las organizaciones necesitan conocer como están desempeñando sus labores los empleados, a fin de identificar quienes efectivamente agregan valor y cuáles no, estos autores van mucho más allá al plantear que hay que discriminar entre empleados efectivos e inefectivos.

Existe la necesidad de saber cómo se están aprovechando los recursos humanos en las organizaciones y de saber si ellos están aportando a la organización y para esto es necesario evaluar su desempeño. Sin embargo, como mencionan Sánchez y Calderón (2012) la evaluación históricamente se restringió al simple juicio unilateral del jefe respecto al trabajo de su empleado pero a medida que ha evolucionado la gestión de recursos humanos, se han establecido generaciones de modelos de evaluación hasta el punto que hoy en día se pueden encontrar modelos de evaluación de cuarta generación.

\section{Implementación del proceso de evaluación del desempeño}

Es deseable que el proceso de implementación para el proceso de evaluación del desempeño siga una estructura claramente preestablecida, en efecto para Fernández, Cubiero y Dalziel (1996) los pasos para evaluar bien el desempeño de los trabajadores son en primer lugar determinar con claridad la razón por la que se desea implementar el proceso, la organización tiene que decidir cuáles son los resultados que necesita obtener por medio del proceso. En segundo lugar, diseñar un proceso adaptado a esas finalidades, es muy probable que dentro de una misma organización el proceso tenga que variar a fin de reflejar las distintas funciones y puestos de trabajo y proporcionar una adecuada formación a todo el personal y por último examinar continuamente el impacto del proceso.

Es necesario consignar que según Pereda, Berrocal y López (2002), existen una serie de principios que se deben respetar a la hora de implantar un programa de evaluación del desempeño. La evaluación del rendimiento es un sistema, no una técnica. Por lo tanto, el método de evaluación se deberá elegir en función de las características de la organización y de los objetivos del sistema. La evaluación del rendimiento no es un sistema disciplinario, siendo uno de sus fines más importantes favorecer el desarrollo de los empleados, por lo que 
el programa se debe plantear como una oportunidad de mejora de las personas y la organización. Todas las personas que deban actuar como evaluadores deberán recibir formación en los objetivos del programa y en las técnicas que se vayan a emplear. Asimismo, es preciso que los empleados, que van a ser evaluados, reciban una formación paralela, tanto en los aspectos de autoevaluación, como en los objetivos del programa y en el sistema que se va a seguir. Todos los implicados se deben comprometer y participar en el sistema de evaluación, por lo que será necesario que los objetivos del programa se hayan definido y comunicado muy claramente con anterioridad. En ningún caso se comparará al evaluado con otros compañeros, ya que el objetivo de la evaluación es conocer el rendimiento de cada empleado, no comparar unos con otros y por último dado que los sistemas de evaluación funcionan en base a una buena comunicación superior/subordinado, consiguen una mayor eficacia cuando en la organización existe un estilo de dirección participativa.

A los empleados se les debe entregar toda la información sobre el proceso completo de implementación del sistema de evaluación, es decir, se les debe informar sobre cuándo se les evaluará, quién los evaluará y cómo se realizará aquello, de modo que no existan sorpresas cuando se esté ejerciendo la evaluación y de que los mismos no estén a la defensiva, Maristany (2007). Uno de los problemas esenciales que ocurren en el proceso, según Werther y Davis (2013) es la comprensión del evaluador del proceso y su congruencia con el sistema adoptado, para lo cual algunas organizaciones han optado por desarrollar manuales detallados en los cuales se describen minuciosamente los métodos y políticas en vigor, pautas para la conducción de las evaluaciones, así como definiciones de parámetros esenciales. También es importante mencionar, que la capacitación de los evaluadores debe ser un proceso continuo, de modo que éste pueda garantizar congruencia y exactitud, en donde se debe también abarcar cómo realizar entrevistas de evaluación y cómo dar y recibir retroalimentación, Mondy y Noe (2005). Otro aspecto a considerar en la implementación es el registro de la información, si bien mantener un registro continuo de incidentes observados y reportados puede ser una tarea tediosa para los supervisores, es imprescindible cuando se desea realizar una evaluación que proporcione información útil, Mondy y Noe (2005). Es necesario que el supervisor informe respecto a las evaluaciones otorgadas a su jefe inmediato, a fin de que exista más de una opinión respecto al desempeño del empleado, Maristany (2007). La documentación de toda la normativa en la implementación es vital, si las normas se basan en conceptos subjetivos, la evaluación requerirá una interpretación y contendrá juicios personales y decisiones menos claras, Harris (1993). Si se han cumplido las normas, el trabajador recibe una recompensa por sus esfuerzos, pueden otorgarse recompensas para apoyar el buen desempeño, algunas veces se aplican sanciones, con el fin de acabar con el desempeño deficiente y de estimular mejoras. El uso de las técnicas de motivación positiva y negativa pueden resultar muy eficaces, si se aplican para influir en el desempeño del trabajador, Harris (1993). 
El uso y manejo de formularios es fundamental para realizar una buena evaluación, una vez realizada la evaluación, los formularios se regresan a recursos humanos, es el tiempo de analizar los contenidos y detectar los conflictos que se manifiestan en la evaluación y acerca de los cuales recursos humanos debería ayudar a las partes. Adicionalmente se deben tomar los datos sobre potencial, carrera, capacitación y remuneraciones, Maristany (2007). Además, el uso y manejo adecuado de formularios es clave para los contralores del proceso y demás personal administrativo, deben recibir la información con el objeto de coordinar los esfuerzos individuales con las necesidades de la empresa, Harris (1993).

Si la empresa no cuenta con un procedimiento de quejas formal, debe desarrollar uno que proporcione a los empleados la oportunidad de confrontar los resultados de la evaluación que consideren inexactos o injustos. Deben tener un procedimiento para presentar sus quejas y que estas sean abordadas objetivamente, Mondy y Noe (2005).

Si además de evaluar el rendimiento se realiza una valoración del potencial, los gráficos de potencial por unidades organizativas también nos aportan información interesante para conocer en qué unidades hay que hacer énfasis para el desarrollo del talento. Esto consistiría en representar un organigrama donde, con un código de colores, pueda identificarse el nivel de potencial alcanzado en cada una de las unidades organizativas, Sastre y Aguilar (2003).

Finalmente, es necesario que antes de la entrevista final de evaluación se entregue una copia de los resultados de la evaluación al trabajador para que la revise antes de la entrevista, en el caso que la organización quiera que el trabajador tenga una reacción elaborada y sin sorpresas, esto por lo menos con un día de anticipación. Maristany (2007), afirma que de esta forma el empleado tendrá tiempo para pensar acerca de la situación, lo cual sirve para una mejor comunicación posterior.

Para entender la complejidad de un proceso de implementación del desempeño, hay bastante experiencia en el ámbito de la educación, principalmente en el sector público, principalmente en Chile, son varios los autores en el área, Assael y Pavez (2008), Horton (1999), Quaas y Crespo (2003), Avalos, B. y Assaél, J. (2006), Inzunza, J. (2008) y Rueda, M., Elizalde, L. y Torquemada, A. (2003). Las dificultades en la implementación del proceso de evaluación académica son múltiples y complicadas de ser abordadas y también de difícil solución Luna y Torquemada (2008), Montenegro (2003) y Zabalza (2003). Una visión que integra el mundo público y el privado es la que relaciona una adecuada implementación del sistema necesariamente con el sistema de compensaciones que tenga la organización, Sánchez, Pizarro, Alvarez, Castillo y Alfaro (2017).

En el ámbito empresarial, la documentación del proceso de implementación de los sistemas de es mucho más escasa, en efecto autores como Siegfried (1965), Alles (2013) y Chiavenato (2017), plantean revisiones exitosas de procesos de implementación de procesos de evaluación del personal. Por su parte otros autores plantean que se deben evaluar muy 
bien los riesgos que tiene asociado el proceso de evaluación en las empresas, Sánchez (2013) y Sánchez y Rojas (2014).

\section{Auditoría y recursos humanos}

Para Pérez y Oreo (2006), la auditoría de recursos humanos busca deficiencias y aporta para mejorar los procesos en la empresa, esto incluye a cada uno de los trabajadores de la organización. La auditoría ayuda a evaluar a cada empleado para ver si es el indicado en el puesto y además revisar qué es lo que puede mejorar para aportar más a su puesto de trabajo (Sanchez y Rojas, 2014). El ámbito de acción de la auditoría de recursos humanos es muy amplio, en efecto, García (2003) plantea que esta auditoría, no se refiere solamente a la formación interna de un equipo de dirección, sino también a sus valores, a su estilo de dirección y sus orientaciones.

Existen otras posturas, como la de Sánchez y Rojas (2014) que define la auditoría de recursos humanos como un proceso de evaluación sistemático que validará las políticas, prácticas y programas de recursos humanos en cuanto a su contribución a los objetivos de la empresa como organización y que concluirá con un informe en el que se recogerán puntos fuertes y débiles de las actividades realizadas, así como indicaciones de mejora. Al realizar una auditoría de recursos humanos se obtienen varios beneficios entre los que figuran el identificar el aporte que hace el departamento de recursos humanos a la organización, se mejora la imagen profesional del departamento de recursos humanos, se alienta al personal de recursos humanos a asumir mayor responsabilidad y actuar en un nivel más alto de profesionalismo, se esclarecen las responsabilidades, los deberes del departamento de recursos humanos, se facilita la uniformidad de las prácticas y políticas de los recursos humanos, se destacan problemas latentes, potencialmente explosivos, se garantiza el cumplimiento de las disposiciones legales, se reducen los costos de recursos humanos mediante prácticas mejoradas, se promueven los cambios necesarios en la organización y se identifican los problemas que tienen una importancia crítica. Dentro de esos problemas está el riesgo de auditoría que se corre al revisar el desempeño en la empresa, como lo plantea claramente Sánchez (2017). En ese sentido la auditoría de recursos humanos se orienta a evaluar la gestión estratégica de los recursos humanos (De Quijano y Navarro, 1999, Sánchez y Bustamante, 2008 y Nevado, 1988), la auditoría de la gestión de recursos humanos abarcan a un triple colectivo; los especialistas en recursos humanos, los directivos de línea y los trabajadores (Alfaro de Prado, A., Rodriguez, L. y Román, M. 1999). Una visión más estratégica de la auditoría de recursos humanos, que considere la interrelación entre los objetivos de la organización con las políticas y estrategias del área de personal, es la que han dado Sánchez Pérez (2014) y Martinez, Fernandez y Tarazona (2016). 
Desde el punto de vista de procesos técnicos, uno de los primeros programas de auditoría publicados aunque muy rudimentario es de origen brasileño (Siegfried,1965), en la misma línea hay un clásico que en sus inicios se atrevió a diseñar un programa de auditoria (Chiavenato, 2017), claramente hay una gran dificultad en el diseño de un programa de auditoría para evaluar recursos humanos y ello se debe al cruce de dos áreas muy diferentes la auditoria y los recursos humanos, una adecuada evaluación por tanto, debe considerar ambos marcos teóricos, en efecto, la construcción del programa de auditoría publicado por Sánchez y Rojas (2014), posee como soporte teórico ambos marcos, uno que viene desde la vertiente de la auditoría clásica con autores que comienzan con Paton (1943), Mautz (1970), hasta obras más contemporáneas como las de Arens, Elder y Beasley (2007), PriceWaterhouseCoopers (2007) y Fonseca (2013), no se puede dejar de mencionar que en el intertanto de esas décadas, resulta importante los aportes en el área de auditoría de Coopers y Lybrand (1984), Slosse y otros (1991), Arens y Loebbecke (1996), Téllez (2004) y Whittington y Pany (2005). En el caso de los aportes en el área de los recursos humanos, pasa algo similar, se comienza con la influencia de algunos autores, en los años ochenta y noventa, tales como Miranda, Torras y González (1982), Bentley (1993), Valle y Weiss (1995), Rodriguez y Ramirez (1997), Dolan, Schuler y Valle (1999), para llegar a influencias más recientes como Werther y Davis (2013), Sánchez (2013), Alles (2014), Sánchez-Perez (2014) y Chiavenato (2017), sin embargo también, es muy importante reconocer los aportes de autores en la década de los años 2000, tal es el caso de Reyes (2002), Sastre y Aguilar (2003), Ariza, Morales y Morales (2004), Mondy y Noe (2005). En esta investigación se utilizó el instrumento de evaluación desarrollado por Sánchez y Rojas (2014) ya que en él se consideran todas las influencias de ambas áreas de trabajo (Auditoría y Recursos Humanos).

La auditoría de recursos humanos se realiza en condiciones de riesgo y por ello es fundamental analizar, evaluar y dimensionar los distintos riesgos que se presentan en su ejecución, estos riesgos deben ser la base de los procedimientos de auditoría, Sánchez y Ramirez (2017). En definitiva, se debe realizar una auditoría estratégica a la función de dirección de personas para ver el adecuado cumplimiento de los objetivos de la organización, Martínez, Fernández y Tarazona (2016). Para cada atributo revisado y evaluado en la auditoría de recursos humanos se debe analizar detalladamente el concepto técnico que puede generar un riesgo al auditor. Sanchez (2019).

En dicho contexto, el presente trabajo tiene como objetivo identificar la estructura en las relaciones entre un grupo de variables asociadas a la "implementación" del proceso de evaluación del desempeño de los trabajadores en diversas organizaciones en Chile. 


\section{Material y Métodos}

\section{Diseño del estudio}

Es un estudio analítico, observacional de cohorte transversal de 116 empresas chilenas, públicas y privadas. A nivel de tamaño de las empresas, se distribuyen en 46 empresas grandes, 60 empresas medianas o pequeñas y 10 microempresas. Las empresas en estudio corresponden a los siguientes rubros: Agricultura 5, Minería 1, Manufactura 2, Suministros 21, Construcción 4, Comercio 8 Hoteles y Restaurantes 9, Transporte 4, Intermediación Financiera 10, Servicios Públicos 20, Juegos y Comunicación 2, Enseñanza 21 y Servicios 9 empresas. Del total de 116 organizaciones evaluadas, 25 corresponden a organizaciones públicas y 91 a organizaciones privadas.

Los datos fueron recopilados, directamente por el equipo de trabajo, entre los meses de junio de 2016 y julio de 2017. El análisis estadístico se realizó con SPSS versión 22

\section{Confiabilidad del instrumento}

A partir de un instrumento desarrollado por Sánchez y Bustamante (2008) y que fue revisado por expertos y posteriormente presentado para su revisión en varios congresos internacionales (XLIII Asamblea Anual del Consejo Latinoamericano de Escuelas de Administración, realizada en México el año 2008, CLADEA 2009 realizada en Ecuador y CLADEA 2011 realizada en Puerto Rico). El instrumento se aplicó en todas las etapas del proceso de implementación del sistema de evaluación del desempeño de acuerdo a lo mostrado en la Figura 1.

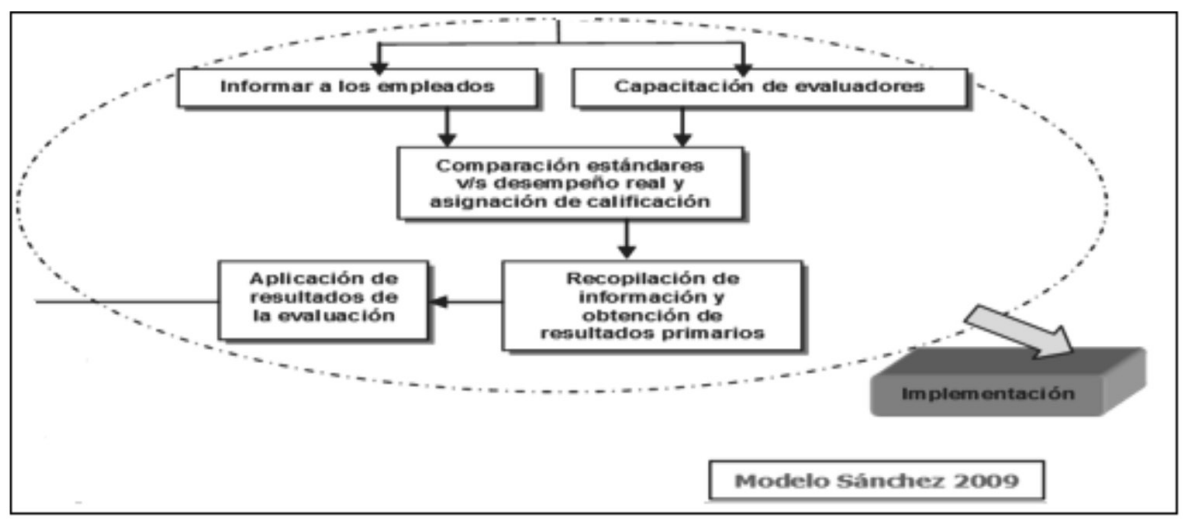

Figura 1.Flujograma del proceso de implementación del desempeño en la organización Fuente: Sánchez, J. (2014). Control de gestión del desempeño de los recursos humanos. Pág. 120. España: Editorial Apyce 
El registro de los datos lo realizó un equipo de auditoría, para disminuir el sesgo, se utilizó un formulario electrónico, que permite prevenir valores inexactos y fueron registrados por personas que no participaron del análisis. El formulario electrónico (Microsoft Office), diseñado a partir del instrumento seleccionado, contiene 22 variables a evaluar.

\section{Definición de Variables}

Los procedimientos realizados, divididos por variables, aplicados a cada una de las 116 empresas, y cuya calificación es la siguiente:

$$
\begin{array}{llll} 
& \multicolumn{4}{c}{\text { 0 -57 }} & \multicolumn{2}{c}{\text { puntos }} & \text { Muy malo } \\
2 & 58-72 & \text { puntos } & \text { Regular } \\
3 & 73-86 & \text { puntos } & \text { Bueno } \\
4 & 87-100 & \text { puntos } & \text { Muy bueno }
\end{array}
$$

VAR01: Información a los empleados: Hacer indagaciones con los responsables del proceso sobre la realización de sesiones informativas para los empleados y a sus representantes. Obtener confirmaciones de los empleados o sus representantes respecto de la realización de las sesiones informativas. Revisión de documentación tipo citaciones, para concurrir a dichas sesiones. Revisión de registros que acrediten la asistencia de los empleados a dichas sesiones. y Solicitar a los empleados que expliquen el sistema de evaluación que se les aplica.

VAR02: Validez de la información: Solicitar a los encargados de entregar la información que especifiquen qué tipo de información se les entregó a los empleados y realizar un análisis de sus declaraciones para comprobar que se abarcaron todos los puntos necesarios. Solicitar a los empleados que respondan si conocen los siguientes aspectos del sistema, los cuales debieron abarcar en la sesión informativa, para verificar que son conocidos por ellos (Objetivo que persigue el sistema, Expectativas de desempeño, Dimensiones, Escalas utilizadas, Oportunidad y periodicidad de la aplicación, Quiénes serán sus evaluadores, Qué evaluará cada uno de ellos, Posibles acciones y consecuencias según los resultados obtenidos y Cuándo comienza su aplicación). Verificar que se les entregó un documento informativo de evaluación a los empleados, mediante la observación del documento o la confirmación de entrega por parte de los empleados. Comparar la información conocida por los empleados con la entregada por los dirigentes del proceso.

VAR03: Capacitación a evaluadores: Indagar con los responsables del proceso, si se realizó una capacitación para los evaluadores. Confirmar la realización de dicha capacitación con los evaluadores. Revisión de citaciones escritas enviadas a los evaluadores invitándoles a participar en estas capacitaciones. Revisión de documentos que acrediten gastos referidos a la realización de dichas capacitaciones, por ejemplo, arriendos de establecimientos, gastos 
de equipamientos, alimentación, salario a los capacitadores, etc. y revisión de registros que acrediten la asistencia de los empleados a estas capacitaciones.

VAR04: Oportunidad de la capacitación: Verificar que la capacitación se realizó antes del período de evaluación, a través de inspección de registros e indagaciones con el personal.

VAR05: Validez de la capacitación: Indagar con los evaluadores para determinar el conocimiento de los aspectos relevantes del proceso de evaluación, relacionados con aspectos relevantes (Explicación del objetivo que persigue el sistema, Dimensiones, Escalas utilizadas, Expectativas de desempeño, Oportunidad y periodicidad de la aplicación, Cómo aplicar la evaluación, respecto a los parámetros, Posibles problemas y forma de superarlos, Cómo realizar entrevistas y entregar retroalimentación, Posibles acciones y consecuencias, según los resultados obtenidos, Cuándo comienza su aplicación y Realización de ejercitaciones de evaluación, y utilización de instrumentos). Verificar la entrega de un manual de evaluación que contenga todos los aspectos relevantes del proceso explicados en detalle, mediante la observación de dicho manual. Evaluar la experticia y/o conocimiento del tema, del personal que realizó la capacitación: Verificar el currículum, Verificar que tiene experiencia en el tema de evaluación del desempeño, Verificar que tiene experiencia en capacitaciones anteriores en la empresa y Verificar la realización de actividades anteriores relacionadas con el tema.

VAR06: Simetría de información: Realizar una comparación entre la información conocida por los empleados y la información conocida por los evaluadores del sistema y verificar que concuerdan.

VAR07: Aceptación del sistema y sus ventajas por parte de evaluadores: Realizar indagaciones con los evaluadores para determinar si estos consideran que el sistema es efectivo y contribuye a mejorar el desempeño, Revisión de formularios u otros registros que evidencien el uso del sistema por los evaluadores, Revisión de evaluaciones históricas para determinar la evolución del desempeño e Indagar con el personal respecto al tiempo dedicado a la realización de evaluaciones.

VAR08: Existencia y documentación de supervisión continua: Revisión de los documentos o archivos que contengan registros de las supervisiones o del desempeño de los empleados, Verificar la existencia de reportes entregados por parte de los trabajadores a sus superiores y determinar mediante la existencia de anotaciones o firmas la revisión de los mismos, Revisión de las fechas registradas en los documentos, verificar que éstas son periódicas, Indagación de los registros, para verificar que la documentación de la supervisión es realizada, Revisión de los archivos, para verificar que se está utilizando el método complementario definido por la empresa, Indagar con los empleados sobre la realización de supervisión o revisión del desempeño y Observación de cartas o documentos similares, en donde se informe al empleado su desempeño, en períodos anteriores al establecido para la evaluación formal y en el período actual. 
VAR09: Realización de entrevistas: Realizar indagaciones con los trabajadores, para verificar si reciben retroalimentación constante, Para una muestra representativa de trabajadores, identificar deficiencias o calificaciones sobresalientes en las evaluaciones y consultar al supervisor cuándo percibió dichas deficiencias o desempeño excelente. Luego consultar con los empleados involucrados cuándo se les dio a conocer personalmente por su jefe y determinar el tiempo transcurrido entre ambos sucesos y Revisión de registros informales que acrediten encuentros entre supervisores y evaluados y que contengan, por ejemplo, los temas tratados en esas reuniones.

VAR10: Congruencia entre los objetivos del evaluador y los de la organización: Indagar con los evaluadores sobre el propósito para el cual ellos realizan las evaluaciones y comparar lo que el evaluador persigue y el objetivo del sistema declarado por la organización, Indagar con los evaluados sobre su opinión del objetivo perseguido por el evaluador y Realizar indagaciones para determinar cuáles son las conclusiones de las entrevistas y verificar si dichas conclusiones se relacionan con el objetivo planteado por la organización.

VAR11: Documentación evaluación formal: Revisar la documentación o archivos correspondientes a las evaluaciones formales.

VAR12: Uniformidad en la aplicación de los estándares del cargo: Conocer los estándares, Revisar las evaluaciones realizadas a una muestra de empleados en un mismo cargo y ver la aplicación de dichos estándares y Realizar cálculos independientes de las calificaciones que se debieron otorgar a los empleados de un mismo y verificar que éstas corresponden a las otorgadas por el evaluador.

VAR13: Revisión de la evaluación: Seleccionar una muestra de las evaluaciones y analizarlas en busca de evidencia de la revisión e Indagar con los superiores del evaluador respecto a la revisión de las evaluaciones otorgadas por él.

VAR14: Exactitud de la calificación: Para una muestra, rehacer los cálculos matemáticos.

VAR15: Entrega oportuna de la información: Indagar con el trabajador la fecha de entrega de las mismas y compararla con la establecida para realizar la entrega y Revisar la firma del empleado en su hoja de evaluación u observaciones, como cumplimiento a un procedimiento administrativo de conformidad.

VAR16: Entrega de copia de evaluación al empleado: Verificar que a los empleados se les entregó una copia de su evaluación y Comprobar que se entregó la copia con la antelación suficiente para permitir que la analicen antes de la entrevista.

VAR17: Apelaciones: Obtener una descripción del procedimiento de apelación, Comprobar que todo el personal evaluado pueda decidir si apela o no el resultado de su evaluación e Identificar empleados que apelaron y realizar un seguimiento de sus casos, para determinar si fueron cursados en relación a los procedimientos de apelación. 
VAR18: Eficacia del proceso de apelación: Realizar un seguimiento de evaluaciones que fueron reconsideradas y analizar las causas, Indagar con el trabajador si consideraron efectivo el proceso de apelación e Indagar con el evaluador si consideró pertinente la resolución de la apelación.

VAR19: Aplicación de acciones predefinidas: Identificar cuáles fueron los usos que se les dieron a las evaluaciones y comparar el desempeño con las acciones que se predefinieron y Analizar evaluaciones situadas en los extremos y realizar un seguimiento y evaluar si las acciones seguidas son efectivas.

VAR20: Privacidad e integridad de la información: Determinar la existencia de controles que garanticen el acceso limitado a la documentación y Verificar que las evaluaciones de períodos anteriores (que aun puedan necesitarse), están en poder del departamento de recursos humanos, en un lugar seguro.

VAR21: Suficiencia de recursos y tiempo: Comparar el tiempo total que abarcó el proceso de implementación con el tiempo asignado para ello, Comparar el tiempo de evaluación utilizado por trabajador con el tiempo definido, Realizar indagaciones con el personal respecto a la suficiencia de los recursos y Conciliar los gastos efectivos con los presupuestados.

VAR22: Realización del proceso de evaluación: Comparar la realización del proceso, con la planificación realizada por la organización respecto a los varios ítems (Actividades previamente definidas, así como también su secuencia, Tiempo que se utilizó para realizar las diversas actividades, Fechas establecidas, Periodicidad de las evaluaciones y Personal responsable). Satisfacerse que se hayan evaluado a todos los trabajadores, a través de cotejar la planilla de remuneraciones del período con la cantidad de evaluaciones realizadas y con los registros de asistencia y Para una muestra de trabajadores, satisfacerse de que el trabajador evaluado existe, a través de pruebas de indagación y observación, además que prestó sus servicios en el período bajo evaluación.

Los datos analizados en esta investigación son de fuente primaria, recogidos mediante técnicas de análisis documental, la observación directa no participativa y el análisis de los procesos. De un total de 116 empresas evaluadas, se incluyeron 105, que cumplieron los criterios de inclusión, representando un $91 \%$ del total. Los criterios de inclusión se relacionan principalmente con que estuviera completa toda la información solicitada en el instrumento aplicado.

\section{Análisis Factorial}

Se analizaron los procedimientos de auditoría aplicados a partir de análisis factorial exploratorio. Es decir, encontrar aquellos factores que explicaran el máximo de variabilidad y que se estructuraran con variables (ítems) específicas al factor. (Vivanco, 1999; Garmendia, 2007). 
En primer lugar, se realizó un análisis de la matriz de correlaciones. Para el análisis de los valores y vectores propios de la matriz de correlación, se utilizaron 105 casos, 11 casos contienen valores faltantes. La confiabilidad del instrumento se evaluó a través del análisis de su consistencia interna por cálculo del coeficiente del alpha de Cronbach. Un alpha igual o mayor a 0,7 fue considerado como satisfactorio.

\section{Generación de la Matriz de Correlaciones}

Se obtuvo una matriz de las correlaciones entre las 22 variables en estudio y se aplicaron dos pruebas estadísticas: Coeficiente Kaiser - Meyer - Olkin (KMO) con un nivel de aceptación superior a 0,5 y el Test de Esfericidad de Bartlett con un nivel de significación aceptable menor al 5\%, para evaluar la pertinencia de llevar a cabo un análisis factorial.

\section{Extracción de Factores}

El método usado para efectos de extraer los factores iniciales de la matriz de autocorrelación es el método de Análisis de Componentes Principales. Los nuevos factores serán una combinación lineal de las variables originales, y no se correlacionan entre ellos. El modelo busca, en primer lugar, el factor que explica la mayor cantidad de la varianza en la matriz de correlación y en segundo lugar, una combinación línea, la cual busca explicar la proporción máxima de la varianza remanente. Se incorporar factores cuya varianza es mayor que 1. (Garmendia, 2007). Se buscan combinaciones lineales del tipo:

$$
Y_{k}=a_{k 1} x_{1}+\ldots+a_{k p} x_{p}=\sum a_{k j} x_{j}, k=1, \ldots, p,
$$

\section{Cálculo de las comunalidades}

Se determina según el cálculo del coeficiente de determinación múltiple al cuadrado y toma valores entre 0 y 1 . Si un factor tiene un bajo valor propio, entonces está contribuyendo poco a la explicación de la varianza de la variable. (Garmendia, 2007)

\section{Determinación del número de factores}

Para efectos de estudio se tomarán aquellos factores cuyo valor propio sea mayor que 1 y, se complementa, a través, del gráfico de sedimentación que muestra la forma en que van disminuyendo los valores propios, seleccionando el número de factores correspondiente al punto en que la curva del gráfico se hace horizontal. (Garmendia, 2007)

\section{Rotación de los factores}

Se usó el sistema ortogonal, que mantiene la independencia entre los factores rotados, y entre los métodos que ofrece este sistema se aplicó el varimax, generando una matriz de 
componentes rotados que indica la correlación existente entre cada una de las variables y su correspondiente factor. (Garmendia, 2007)

\section{Evaluación del ajuste del modelo}

Para ello se comparará la matriz de correlación inicial con la matriz generada a partir de las variables latentes. Se interpreta los factores resultantes asignándoles nombre considerando las variables originales incluidas en cada factor. (Garmendia, 2007).

\section{Resultados y Discusión}

Se obtuvo un alpha de Cronbach de 0,884 , en tanto se comprueba la confiabilidad del instrumento aplicado. El test de adecuación de la muestra (KMO) es de 0,808, cada variable es perfectamente predicha por las otras variables, es decir, la relación entre las variables es alta. En concordancia con lo anterior, el test de esfericidad de Bartlett es significativo $(\mathrm{p}=$ 0,000), de manera que se confirma la adecuación para el uso del análisis factorial. También, en la matriz de correlaciones, de las 462 correlaciones, 272 (59\%) son significativas al nivel del 0,01 . Tabla 1 .

Tabla 1

Prueba de KMO y Bartlett

Medida Kaiser-Meyer-Olkin de adecuación de muestreo (KMO)

, 808

Prueba de esfericidad de Bartlett Aprox. Chi-cuadrado

1481,823

gl

231

Sig.

, 000

En tabla 2 se muestra los 6 factores, extraídos de la matriz inicial y usando el método de componentes principales. Estos seis factores presentan un valor propio superior a 1. El factor 1 representa el 35,19\% de la varianza y los seis factores, en general, representan el 72,6\% de la variabilidad total, lo que sugiere que dichos seis componentes explicarían adecuadamente la variabilidad de los datos. 
Tabla 2

Porcentaje de varianza total explicada

\begin{tabular}{|c|c|c|c|c|c|c|}
\hline \multirow[b]{2}{*}{ Componente } & \multicolumn{3}{|c|}{ Autovalores iniciales } & \multicolumn{3}{|c|}{ Sumas de extracción de cargas al cuadrado } \\
\hline & Total & $\%$ de varianza & $\%$ acumulado & Total & $\%$ de varianza & $\%$ acumulado \\
\hline 1 & 7,744 & 35,198 & 35,198 & 7,744 & 35,198 & 35,198 \\
\hline 2 & 2,379 & 10,814 & 46,012 & 2,379 & 10,814 & 46,012 \\
\hline 3 & 1,980 & 9,000 & 55,012 & 1,980 & 9,000 & 55,012 \\
\hline 4 & 1,696 & 7,710 & 62,722 & 1,696 & 7,710 & 62,722 \\
\hline 5 & 1,159 & 5,267 & 67,989 & 1,159 & 5,267 & 67,989 \\
\hline 6 & 1,021 & 4,641 & 72,630 & 1,021 & 4,641 & 72,630 \\
\hline 7 &, 839 & 3,812 & 76,443 & & & \\
\hline 8 &, 824 & 3,744 & 80,186 & & & \\
\hline 9 &, 726 & 3,301 & 83,487 & & & \\
\hline 10 & ,656 & 2,983 & 86,470 & & & \\
\hline 11 &, 478 & 2,175 & 88,645 & & & \\
\hline 12 &, 437 & 1,988 & 90,633 & & & \\
\hline 13 &, 376 & 1,710 & 92,343 & & & \\
\hline 14 &, 330 & 1,502 & 93,845 & & & \\
\hline 15 &, 302 & 1,374 & 95,219 & & & \\
\hline 16 &, 233 & 1,060 & 96,279 & & & \\
\hline 17 &, 217 & ,986 & 97,264 & & & \\
\hline 18 & ,166 &, 755 & 98,019 & & & \\
\hline 19 & ,138 & ,629 & 98,648 & & & \\
\hline 20 &, 126 &, 573 & 99,221 & & & \\
\hline 21 & ,106 &, 480 & 99,701 & & & \\
\hline 22 & ,066 & ,299 & 100,000 & & & \\
\hline
\end{tabular}

Fuente: Datos de la investigación. SPSS.

La gráfica de sedimentación, o de valores propios, presenta una curva pronunciada hasta el punto 6 (eje número de factores) y luego continúa una línea recta, por ello, según este método también se infiere seis componentes principales. Figura 2. 


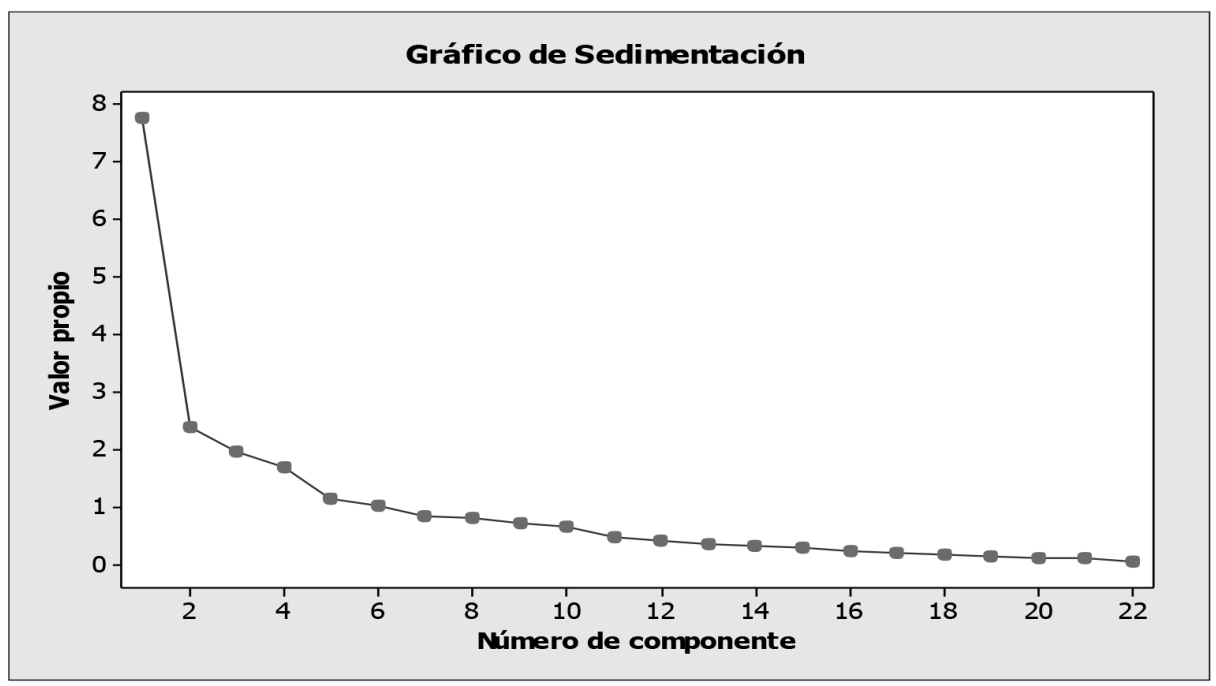

Figura 2. Gráfico de sedimentación de Cattell (1966), o de valores propios.

Fuente: Datos de la investigación. SPSS

Para los datos sobre la implementación de sistemas de evaluación de personal en empresas de Chile, se define la matriz de componente, usando el método de extracción de componentes principales. El componente 1 presenta variables que tienen alta saturación en cada factor Tabla 3.

Tabla 3

Matriz de componentes

\begin{tabular}{|c|c|c|c|c|c|c|}
\hline & \multicolumn{6}{|c|}{ Componentes } \\
\hline & 1 & 2 & 3 & 4 & 5 & 6 \\
\hline VAR00001 &, 723 & ,001 &,- 440 &,- 019 &,- 088 &,- 071 \\
\hline VAR00002 & ,774 &,- 003 &,- 388 &,- 063 &,- 156 &,- 165 \\
\hline VAR00003 &, 528 &,- 716 & ,200 &, 088 &,- 020 &, 037 \\
\hline VAR00004 &, 507 &,- 784 &, 159 &, 084 &, 084 &,- 003 \\
\hline VAR00005 &, 577 &,- 718 &, 160 &, 134 &,- 123 &,- 043 \\
\hline VAR00006 & ,742 & ,082 &,- 369 &,- 189 &,- 007 & ,006 \\
\hline VAR00007 & ,848 & ,169 &, 175 &,- 169 &, 023 &,- 024 \\
\hline VAR00008 & ,605 & ,398 & ,296 &,- 029 &,- 054 &, 043 \\
\hline VAR00009 & ,493 & ,195 &,- 100 &, 047 &,- 144 & ,690 \\
\hline VAR00010 & ,740 &, 061 &,- 124 &,- 189 &,- 109 & ,182 \\
\hline
\end{tabular}




\begin{tabular}{lllllll} 
VAR00011 &, 656 &, 352 &, 229 &,- 057 &,- 098 &,- 317 \\
VAR00012 &, 347 &,- 111 &,- 001 &,- 402 &, 698 &, 248 \\
VAR00013 &, 630 &, 358 &, 338 &,- 063 &,- 103 &,- 175 \\
VAR00014 &, 296 &, 224 &, 475 &,- 140 &, 532 &,- 134 \\
VAR00015 &, 798 &, 076 &,- 163 &, 002 &, 222 &, 077 \\
VAR00016 &, 700 &,- 133 &,- 007 &, 068 &,- 105 &,- 127 \\
VAR00017 &, 248 &, 249 &,- 241 &, 725 &, 226 &,- 258 \\
VAR00018 &, 148 &, 079 &, 084 &, 816 &, 258 &, 121 \\
VAR00019 &, 479 &, 156 &, 486 &, 381 &,- 166 &, 334 \\
VAR00020 &, 526 &, 111 &, 396 &,- 053 &,- 220 &,- 037 \\
VAR00021 &, 293 &, 188 &,- 574 &, 146 &, 053 &, 117 \\
VAR00022 &, 700 &,- 152 &,- 241 &, 034 &, 148 &,- 183 \\
\hline
\end{tabular}

Fuente: Datos de la investigación. SPSS.

Se realizó la rotación de ejes con el objetivo de seleccionar una solución más sencilla e interpretable. Se usó el método de rotación Varimax, permitiendo reducir el número de variables que tienen alta saturación en cada factor. El método de normalización usado fue Kaiser. La rotación convergió en seis iteraciones. Tabla 4.

Tabla 4

Matriz de componente rotados

\begin{tabular}{|c|c|c|c|c|c|c|}
\hline & \multicolumn{6}{|c|}{ Componente } \\
\hline & 1 & 2 & 3 & 4 & 5 & 6 \\
\hline VAR00001 & ,809 & , 186 &, 183 &, 045 &,- 025 & ,066 \\
\hline VAR00002 &, 819 & ,284 &, 218 &,- 009 &,- 071 &,- 002 \\
\hline VAR00003 & ,132 &, 095 &, 896 &,- 003 &, 074 &, 076 \\
\hline VAR00004 &, 145 &, 017 & ,931 & ,019 &, 150 &, 004 \\
\hline VAR00005 & ,198 & ,135 &, 921 & ,016 &,- 045 &, 033 \\
\hline VAR00006 & ,784 & ,253 & ,108 &,- 075 & ,133 &, 122 \\
\hline VAR00007 &, 459 & ,682 &, 213 &,- 035 &, 252 & ,148 \\
\hline VAR00008 & ,208 & ,708 &,- 030 &, 073 &, 126 &, 228 \\
\hline VAR00009 &, 341 &, 171 & ,009 &, 028 &, 051 & ,800 \\
\hline VAR00010 &, 586 &, 370 & ,190 &,- 134 &, 110 &, 318 \\
\hline VAR00011 &, 326 &, 773 &, 017 &, 058 &, 031 &,- 107 \\
\hline VAR00012 & ,222 &,- 023 &, 137 &,- 133 &, 862 &, 110 \\
\hline
\end{tabular}




\begin{tabular}{lcccccc} 
VAR00013 &, 217 &, 794 &, 026 &, 036 &, 066 &, 029 \\
VAR00014 &,- 125 &, 491 &,- 003 &, 117 &, 628 &,- 137 \\
VAR00015 &, 635 &, 331 &, 201 &, 171 &, 328 &, 186 \\
VAR00016 &, 461 &, 386 &, 412 &, 086 &,- 018 &, 027 \\
VAR00017 &, 299 &, 072 &,- 082 &, 836 &,- 076 &,- 144 \\
VAR00018 &,- 066 &, 031 &, 113 &, 852 &, 019 &, 195 \\
VAR00019 &,- 103 &, 566 &, 238 &, 328 &,- 056 &, 525 \\
VAR00020 &, 075 &, 649 &, 218 &,- 060 &,- 031 &, 137 \\
VAR00021 &, 600 &,- 144 &,- 173 &, 221 &,- 016 &, 167 \\
VAR00022 &, 635 &, 200 &, 352 &, 148 &, 174 &,- 092 \\
\hline
\end{tabular}

Fuente: Fuente: Datos de la investigación. SPSS.

El criterio para efectos de seleccionar los ítems en cada factor, es un nivel de saturación superior a 0,7 (Garmendia, 2007). Dicho nivel de saturación se presenta en la matriz de componentes rotadas, y con los ítems seleccionados se procedió a etiquetar a cada uno de los seis factores principales: A nivel de análisis de los componentes principales se tiene lo siguiente:

- Para PC 1 se infiere la variable "Información", el cual presenta una varianza de 7,7437 y explica el $35,2 \%$ de la varianza total en los datos. Se observa que tiene mayor correlación positiva con información a los empleados $(0,8)$, validez de la información $(0,81)$, simetría de la información $(0,78)$, y existe baja correlación con el resto de las variables.

- Para PC 2 corresponde la variable "Evaluación", el cual presenta una varianza de 2,3790 , y explica el 10,8\% de la varianza total en los datos. Se observa que tiene mayor correlación positiva con la variable existencia y documentación de supervisión continua $(0,70)$, documentación evaluación formal $(0,77)$, revisión de la evaluación (0.79) y existe baja correlación con el resto de las variables.

- PC 3 corresponde la variable "Capacitación”, el cual presenta una varianza de 1,9800 y explica el $9 \%$ de la varianza total en los datos. Se observa que tiene mayor correlación positiva con las variables capacitación a evaluadores $(0,89)$, oportunidad de la capacitación $(0,93)$, valides de la capacitación $(0,92)$, y existe muy baja correlación con el resto de las variables.

- PC 4 corresponde la variable “Apelación”, el cual presenta una varianza de 1,6962 y explica el 7,7\% de la varianza total en los datos. Se observa que tiene mayor correlación positiva con las variables apelación $(0,83)$, eficiencia del proceso de apelación $(0,85)$, y existe muy baja correlación con el resto de las variables. 
- PC 5 corresponde la variable "Retroalimentación", el cual presenta una varianza de 1,1588 y explica el 5,3\% de la varianza total en los datos. Se observa que tiene mayor correlación positiva con la variable realización de entrevistas $(0,86)$ y existe muy baja correlación con el resto de las variables.

- PC 6 corresponde la variable “Aplicación”, el cual presenta una varianza de 1,0210 y explica el 4,6\% de la varianza total en los datos. Se observa que tiene mayor correlación positiva con la variable uniformidad en la aplicación de los estándares del cargo $(0,8)$, y existe muy baja correlación con el resto de las variables.

Los ítems 9, 10, 16 y 19 son los que obtuvieron una baja discriminación entre los seis factores.

Se puede inferir que con la identificación de dicho componente para cada uno de los procesos de la evaluación de desempeño de los trabajadores, se podría llegar a construir un índice que permitiera medir el impacto que tendría en la gestión de la empresa la evaluación de desempeño de los trabajadores. Por ello, el presente trabajo contribuye en dicha línea de trabajo, con la identificación de los factores para el primer proceso "Implementación". Los autores Siegfried (1965), Alles (2013) y Chiavenato (2017) plantean que la documentación del proceso de implementación de los sistemas es mucho más escasa, a nivel empresarial. En la misma línea los autores Manjarrés, Castell y Luna (2013) y Kehoe y Wright (2013), plantea que definición estratégica y posterior seguimiento es fundamental. Por ello, el presente trabajo contribuye con la puesta en marcha de la etapa de "implementación" de procesos de evaluación del personal.

\section{Conclusiones}

Se estudiaron 22 variables que se cubrieron con la aplicación de 61 procedimientos de auditoría específicos, los cuales se orientaron a evaluar el proceso completo de implementación de la evaluación del desempeño de los trabajadores.

Los factores identificados en el proceso de implementación del proceso de evaluación del desempeño de los trabajadores en el presente estudio son: Información, Evaluación, Capacitación, Apelación, Retroalimentación y Aplicación. Dichos componentes son fuente para construir un índice que permita medir la evaluación de desempeño de los trabajadores en la gestión de la empresa.

Dichos factores podrían ser medidos transversalmente en empresas pertenecientes a los rubros Educación y cultura, Instituciones sin fines de lucro, Servicios públicos, Retail, Servicios, Transporte, Financiero, Agrícola, Construcción e inmobiliaria, Salud, Productoras y procesadoras de carnes y Automotriz. 


\section{Referencias}

Alfaro de Prado, A., Rodriguez, L. y Román, M. (1999). El factor humano en las relaciones laborales. Manual de dirección y gestión. Madrid: Ed. Pirámide.

Alles, M. (2014). Dirección Estratégica de Recursos Humanos. Buenos Aires: Granica

Alles, M. (2013). Dirección Estratégica de Recursos Humano: Casos Volumen I. Buenos Aires: Granica

Arens, A., y Loebbecke, J. (1996). Auditoria: Un Enfoque Integral ( $6^{a}$ Edición) .México: Prentice Hall Hispanoamericana.

Arens, A., Elder, R. y Beasley, M. (2007). Auditoria: Un enfoque integral (11 ${ }^{a}$ Edición). México: Pearson Prentice Hall.

Ariza, J., Morales, A., y Morales, E. (2004). Dirección y administración integrada de personas, fundamentos, procesos y técnicas en práctica. España: Mc Graw Hill.

Assaél, J. y Pavez, J. (2008). La construcción e implementación del sistema de evaluación del desempeño docente chileno: principales tensiones y desafíos. Revista Iberoamericana de Evaluación Educativa. Volumen 1, Número 2.

Avalos, B.; Assaél, J. (2006). Moving from resistance to agreement: The case of the Chilean teacher performance evaluation. International Journal of Educational Research, 45.

Bentley, T. (1993). Capacitación empresarial. Santa fé de Bogotá, Colombia: McGraw-Hill. Bock, L. (2015). La nueva fórmula del trabajo: Revelaciones de Google que cambiarán su forma de vivir y liderar. Madrid: Penguin Random House Grupo Editorial España.

Coopers y Lybrand. (1984). Manual de Auditoria ( $3^{a}$ Edición). Bilbao: Ediciones Deusto.

Chiavenato, I. (2017). Administración de recursos humanos. El capital humano de las organizaciones (10 Edición). España: McGraw Hill Interamericana.

De Quijano, S. y Navarro, J. (1999). Auditoría del sistema humano, los modelos de calidad y la evaluación organizativa. Revista de Psicología General y Aplicada. 52 (2), 301-328

Dessler, G., y Varela, R. (2011). Administración de recursos humanos: enfoque latinoamericano $\left(5^{a}\right.$ Edición). México: Pearson Educación.

Dolan, S., Valle, R., Jackson, R. y Schuler, R. (2007). La gestión de recursos humanos. (3 ${ }^{a}$ Edición). España: McGraw Hill Interamericana.

Dolan, S., Schuler, R. y Valle, R. (1999). La gestión de recursos humanos. España: McGraw Hill.

Fernández, G., Cubiero, J., \& Dalziel, M. (1996). Las competencias: clave para una gestión integrada de los recursos humanos ( $2^{a}$ Edición). Bilbao: Ediciones Deusto

Fonseca, O. (2013). Sistemas de control interno. Lima, Ediciones IICO.

Fombrun, C., Tichy, N. y Devanna, M. (1984). Strategic human resource management. New York: John Wiley and Sons.

Garcia, J. (2003). Auditoría sociolaboral. Barcelona: Universitat Oberta de Catalunya.

Gómez-Mejía, L., Balkin, D. y Cardy, R. (2008). Dirección y gestión de recursos humanos $\left(5^{a}\right.$ Edición). España: Pearson Educación. 
Gruman, J. y Saks, A. (2011). Performance management and employee engagement. Human Resource Management Review, 21, 123-136.

Garmendia, M. (2007). Análisis factorial: una aplicación en el cuestionario de salud general de Goldberg, versión de 12 preguntas. Rev. Chil Salud Pública. 11 (27), 57-65.

Harris, J. (1993). Administración de Recursos Humanos: Tomo III, concepto de conducta interpersonal y casos. México: Limusa.

Horton, D. (1999). Building capacity in planning, monitoring, and evaluation: Lessons from the field. Knowledge, Technology and Policy. 11(4): 152-188.

Inzunza, J. (2008). La Evaluación Docente en Chile: institucionalización y simulacro. En Julián Gindín (Compilador) Sindicalismo docente en América Latina. Experiencias recientes en Bolivia, Perú, México, Chile y Argentina. Rosario: Ediciones de AMSAFE.

Luna, E. y Torquemada, A. (2008). Los cuestionarios de evaluación de la docencia por los alumnos: balance y perspectivas de su agenda REDIE. Revista Electrónica de Investigación Educativa. Número especial, pp. 1-15

Kehoe, R y Wright, P. (2013). The Impact of High-Performance Human Resource Practices on Employees’ Attitudes and Behaviors. Journal of Management, 39 (2), 366-391.

Kliksberg, B. (1998). Gerencia Pública para la Modernización del Estado. Revista Chilena de Administración Pública, 4 (19), 4-23.

Luecke, R. (2007). Gestión del desempeño. España: Harvard Business School Publishing Corporation, Ediciones Deusto.

Manjarrés, A., Castell, R. y Luna, C. (2013). Modelo de Evaluación del Desempeño Basado en Competencias. Ingeniare, (8) 11-29.

Maristany, J. (2007). Administración de recursos humanos. México: Pearson.

Martínez, À, Fernández, R. y Federico Tarazona, F. (2016). Auditoría estratégica de la función de recursos humanos. España: Editorial Tirant lo Blanch.

Mautz, R. (1970). Fundamentos de Auditoría. Argentina: Macchi.

Milkovich, G. y Boudreau, J. (1994). Dirección y administración de recursos humanos: un enfoque de estrategia. (6 $6^{\text {a }}$ Edición). México: McGraw-Hill.

Miranda, A., Torras, O., González, J. (1982). Auditoría de las empresas socialistas. Tomo I. La Habana: Editorial Combinado Poligráfico Juan Marinello.

Mondy, M., y Noe, R. (2005). Administración de recursos humanos (9 $9^{a}$ Edición). Mexico: Pearson Education.

Montenegro, I. (2003). Evaluación del desempeño docente. Fundamentos, modelos e instrumentos. Bogotá: Ediciones Especiales Magisterio.

Nevado, D. (1998). La auditoría social de los recursos humanos como instrumento de gestión: futuro y limitaciones. Revista de Trabajo y Seguridad Social, (178), 175-230

Parent, D., Sloan, N., y Tsuchida, A. (2015). Tendencias Globales en Capital Humano 2015: Liderando en el nuevo mundo del trabajo. Deloitte University Press.

Paton, W. (1943). Manual del contador. México: UTEHA. 
Pereda, S. y Berrocal, F. (2011). Dirección y gestión de recursos humanos por competencias. Madrid: Editorial Universitaria Ramón Areces.

Pereda, S., Berrocal, F., y López, M. (2002). Gestión de recursos humanos por competencias y gestión del conocimiento. Dirección y Organización, 5 (28). 43-54.

Pérez, R., Leyva C., Bajuelo, A. y Pérez, T. (2015). La evaluación del desempeño individual, una herramienta para la toma de decisiones. Revista Correo Científico, 19 (4), 1-11.

Pérez, E, y Oreo, L., (2006). Manuales de dirección médica y gestión clínica. Madrid: España: Díaz de Santos.

PriceWaterhouseCoopers (2007). Marco Conceptual Metodológico. Enfoque de Auditoria PWC.

Quaas, C. y Crespo, N. (2003). ¿Inciden los métodos de enseñanza del profesor en el desarrollo del conocimiento metacomprensivo de sus alumnos?. Revista Signos, 36(54), 225-234.

Ramlall, S. J. (2006). Identifying and understanding HR competencies and their relationship to organizational practices. Applied H.R.M. Research, 11(1), 27 -38.

Reyes, A. (2002). Relaciones humanas. I Parte. Administración de personal. México: Limusa.

Rodríguez, M. y Ramírez, P. (1997). Administración de la capacitación. México: McGraw Hill.

Rueda, M., Elizalde, L. y Torquemada, A. (2003). La evaluación de la docencia en las universidades mexicanas. Revista de la Educación Superior 127, 71-77.

Salgado, J. F., y Cabal, Á. L. (2011). Evaluación del desempeño en la Administración Pública del Principado de Asturias: Análisis de las propiedades psicométricas. Revista de Psicología del Trabajo y de las Organizaciones, 27(2), 75-91.

Sánchez-Perez, J. (2014). Fundamentos de auditoría de recursos humanos. España: McGraw-Hill Interamericana.

Sánchez, J. (Octubre de 2019). Información para la gestión de la implementación de sistemas de evaluación de personal en empresas chilenas. XXXIII Conferencia Interamericana de Contabilidad, Cartagena de Indias, Colombia.

Sánchez, J. (2017). Propuesta de matrices de riesgo para el desempeño del personal en las organizaciones. Revista Internacional Legis de

Contabilidad y Auditoría. Colombia. (72):143-182.

Sánchez, J. (2014). Control de Gestión del Desempeño de los Recursos Humanos. España: Editorial Apyce.

Sánchez, J. (2013). Control de Gestión del Desempeño de los Recursos Humanos (2a Edición). Chile: Thomson Reuters.

Sánchez, J., Alvear, S. (2018). Planificación del sistema de evaluación del desempeño de recursos humanos: el caso de empresas chilenas.

Revista ENIAC PESQUISA, 7 (2), 161-181. 
Sánchez, J., Pizarro, I., Alvarez, A., Castillo, M. y Alfaro, M. (2017). Auditoria a procesos de compensación en empresas de una economía

emergente. Revista ENIAC PESQUISA. 6 (2), 247-270.

Sánchez, J. y Ramirez, K. (2017). Análisis del Riesgo en el Mercado Laboral. Parte II. Revista Contabilidad y Auditoría. (46): 111-152.

Sánchez. J. y Rojas, O. (2014). Auditoría de recursos humanos. Madrid: Apyce.

Sánchez, J. y Calderón, V. (2012). Diseño del proceso de evaluación del desempeño del personal y las principales tendencias que afectan su auditoría. Revista Pensamiento y Gestión. Colombia. Enero-Junio (32): 54-82.

Sánchez, J., Bustamante, K. (2008). Auditoría al Proceso de Evaluación del Desempeño. Revista Contabilidad y Auditoría, (198), 105-133.

Sastre, M., y Aguilar, E. (2003). Dirección de Recursos Humanos, un enfoque estratégico. Madrid: McGraw Hill.

Siegfried, H. (1965). Auditoria de Pessoal. RAE-Revista de Administracao de Empresas. 5(17), 149-168.

Slosse, C., Giordano, S., Servideo, F., Dreispiel, G., Pace, C., y De marco, D. (1991). Auditoria: Un Enfoque Empresarial. Buenos Aires: Ediciones Machi.

Téllez, B. (2004). Auditoría un enfoque práctico. México, Thomson.

Trillo Holgado, M. A. (2001). La evaluación del desempeño: nuevas tendencias e implicaciones. Capital Humano, 14(150), 66-72.

Ulrich, D. y Brockbank, W. (2007). La propuesta de valor de los recursos humanos. Barcelona: Deusto S.A. Ediciones.

Valdés, M., Garza, R., Pérez, I., Gé, M., y Chávez, A. (2015). Una propuesta para la evaluación del desempeño de los trabajadores apoyada en el uso de técnicas cuantitativas. Ingeniería Industrial, 36(1), 48-57.

Valle, R. y Weiss, D. (1995). La Gestión estratégica de los recursos humanos. Delaware: Addison-Weasly Iberoamericana.

Valle, R. (2004). La gestión estratégica de los recursos humanos ( $2^{\circ}$ Edición). Madrid, España: Pearson Educación.

Werther, W. y Davis, K (2013). Administración de recursos humanos, el capital humano de las empresas ( $7^{a}$ Edición). México: McGraw-Hill Interamericana.

Wittington, O. y Pany, K. (2005). Principios de auditoría (14 ${ }^{a}$ Edición). México: McGraw-Hill Interamericana.

Wright, P., Dunford, B. y Snell, S. (2001). Human resources and the resource based view of the firm. Journal of Management, 27, 701-721.

Zabalza, M. (2003). Competencias docentes del profesorado universitario. Calidad y desarrollo profesional. Madrid: Narcea. 